\title{
Uncovering the Mechanisms of Conscious Face Perception: A Single-Trial Study of the N170 Responses
}

\author{
Joaquin Navajas, ${ }^{1}$ Maryam Ahmadi, ${ }^{1}$ and Rodrigo Quian Quiroga ${ }^{1,2}$ \\ ${ }^{1}$ Centre for Systems Neuroscience, University of Leicester, Leicester LE1 7RH, United Kingdom, and ${ }^{2}$ Leibniz Institute for Neurobiology, University of \\ Magdeburg, D 39118 Magdeburg, Germany
}

When a face is flashed to an observer, a large negative component is elicited in the occipitotemporal cortex at $\sim 170 \mathrm{~ms}$ from the onset of presentation (N170). Previous studies have shown that the average N170 is correlated with conscious face perception; however, the single-trial mechanisms underlying such modulation remain largely unexplored. Here, we studied in human subjects the average and the single-trial N170 responses to briefly flashed faces, coupled with backward masking and varying degrees of Gaussian noise. In the average evoked responses we observed that, at fixed levels of noise, supraliminal faces exhibited significantly larger N170 amplitudes than subliminal faces. Moreover, the average N170 amplitude decreased with noise level both for the perceived and the nonperceived faces. At the single-trial level, the N170 amplitude was modulated by conscious recognition, which allowed predicting the subjects' perceptual responses above chance. In contrast, the single-trial N170 amplitudes were not modulated by the amount of noise and the effect found in the average responses was due to different latency jitters, as confirmed with latency-corrected averages. Altogether, these results suggest that conscious face perception is correlated with a boost in the activity of face-selective neural assemblies, whereas the stimulus uncertainty introduced by the added noise decreases the timing consistency (but not the amplitude) of this activation.

\section{Introduction}

Previous studies aimed at identifying the neural correlates of face perception have reported the existence of an event-related potential (ERP) in the occipitotemporal cortex, which is larger for faces than other objects, at $\sim 170 \mathrm{~ms}$ from the onset of stimulus presentation (N170) (Bentin et al., 1996). The types of stimuli and conditions that elicit the N170 response have been extensively studied (for review, see Eimer, 2011; Rossion and Jacques, 2011), along with its modulation by attention (Furey et al., 2006; Landau et al., 2007; Engell and McCarthy, 2010) and visual expertise (Rossion et al., 2002; Gauthier et al., 2003; McKone et al., 2007), among other factors.

Over the past years, several studies have reported that the average N170 is correlated with conscious face perception (Fisch et al., 2009; Harris et al., 2011; Rodríguez et al., 2012). However, with one exception (Fisch et al., 2009; see Discussion), previous works reporting differences between perceived and nonperceived faces induced lack of awareness by changing physical properties of the stimuli, such as the time of presentation (Liddell et al., 2004; Genetti et al., 2009; Pegna et al., 2011), the temporal sepa-

Received March 12, 2012; revised Nov. 7, 2012; accepted Nov. 22, 2012.

Author contributions: J.N. and R.Q.Q. designed research; J.N. and M.A. performed research; J.N., M.A., and R.Q.Q. analyzed data; J.N. and R.Q.Q. wrote the paper.

This work was funded by the Engineering and Physical Sciences Research Council, UK. We thank the Psychological Image Collection at Stirling (http://pics.psych.stir.ac.uk/) for providing facial stimuli, and Kaliilah Bolkiah for assistance in data collection.

The authors declare no competing financial interests.

Correspondence should be addressed to Joaquin Navajas, Centre for Systems Neuroscience, Engineering Building, University of Leicester, University Road, Leicester LE1 7RH, UK. E-mail: jmna1@le.ac.uk.

DOI:10.1523/JNEUROSCI.1226-12.2013

Copyright $\odot 2013$ the authors $\quad 0270-6474 / 13 / 331337-07 \$ 15.00 / 0$ ration to a mask (Bacon-Macé et al., 2005; Harris et al., 2011), the stimulus uncertainty given by its phase coherence (Philiastides and Sajda, 2006) or the amount of noise added to the faces (Jemel et al., 2003). These studies have provided valuable insights on how the N170 correlates with face perception, but have the caveat that the effect of conscious face perception is mixed up with the one given by processing stimuli with different physical properties. Moreover, the single-trial underpinnings of the N170 modulation by conscious face perception and by stimulus uncertainty remain largely unexplored. This is particularly important considering that an increase in the average ERP could be due to an increase of the amplitude of the single-trial responses, or due to a better time locking to the onset of the stimulus, thus reflecting different mechanisms (Quian Quiroga, 2000; Quian Quiroga and Garcia, 2003; Atienza et al., 2005).

Here, we studied the average and single-trial N170 responses to briefly flashed faces, coupled with backward masking and varying degrees of Gaussian noise. The amount of noise was tuned throughout the experiment using a double-staircase procedure. After completing the experiment, we could determine for each subject three levels of noise at which they failed to recognize the flashed image as a face in $\sim 30 \%, 50 \%$, and $70 \%$ of the trials. This provided us the opportunity to simultaneously assess the effect of conscious face perception using identical stimuli (comparing the N170 for seen and unseen trials at fixed noise levels) and the effect of stimulus uncertainty (comparing the N170 for different levels of noise for each perceptual outcome: recognized or not). Moreover, we implemented a single-trial analysis to further understand the mechanisms underlying these two different modulations. 


\section{Materials and Methods}

Stimuli. Twenty pictures of faces in frontal view were selected from the Psychological Image Collection at Stirling (http://pics.psych.stir.ac. $\mathrm{uk} /$ ), and 20 front views of cars were downloaded from different websites. A mask was created with randomly shuffled pieces taken from different images. All pictures were converted to grayscale and cropped to a resolution of $411 \times 527$ pixels. The mean brightness was normalized across images. Zero-mean Gaussian noise was added to the car and face images, with 10 different degrees of variance, linearly increasing from 0 to 1 , expressed in units relative to the mean brightness of the picture. Pictures were presented in the center of a screen with a gray surrounding background, on a CRT Monitor with a resolution of $1024 \times 768$ pixels and a refresh rate of $70 \mathrm{~Hz}$. The viewing distance was $50 \mathrm{~cm}$.

Paradigm. Each trial (Fig. 1) began with a fixation cross presented for a variable time $(500-700 \mathrm{~ms})$, followed by a picture of a face or a car shown for $57 \mathrm{~ms}$, and a mask that was shown for $443 \mathrm{~ms}$. Participants were instructed to click the left mouse button whenever they saw a face and click the right mouse button otherwise. Throughout the experiment, a double-staircase procedure was implemented-only for face images - by changing the variance of the Gaussian noise. The descending staircase started with a variance of 1 , and the ascending staircase with a variance of 0 (no noise), with a step-size of 0.1 .

Overall, the experiment consisted of 1200 trials, randomly interleaved: 200 corresponded to the presentation of faces without noise, 200 to the presentation of cars without noise, 350 to the ascending staircase, 350 to the descending staircase, and in the remaining 100 trials cars were presented with the level of noise determined by the staircase procedure for the current trial. These last set of "catch trials" were added to assess the reliability of the subjects' reports as established by the number of false positives (i.e., trials in which the participant reported seeing a face when a car was presented). After blocks of 240 trials, subjects were able to take a short rest break. The experiment lasted $\sim 1 \mathrm{~h}$ and $15 \mathrm{~min}$.

After completing the experiment, we selected for each subject a level of noise in which the face-detection performance was $\sim 50 \%$ (i.e., threshold condition). The double-staircase procedure also allowed us to select higher and lower levels of noise in which the performances were $\sim 30 \%$ and $70 \%$, respectively (i.e., sub-threshold and supra-threshold condition). We then separated the trials according to the level of noise and to the subjects' report (seen or unseen), leading to six conditions (i.e., subthreshold unseen, sub-threshold seen, threshold unseen, threshold seen, supra-threshold unseen, and supra-threshold seen).

Participants. Twenty-two participants (mean age 27.9 years; age range 21-37 years; 2 were left handed; 12 females) volunteered for this study. Subjects were students and researchers at the University of Leicester. All of them had normal or corrected-to-normal vision and no known neurological impairments. Data from another six participants were discarded due to an excessive number of false positives $(\geq 10 \%)$ compared with an average of $1.7 \%$ (range $0-5 \%$ ) for the remaining 22 subjects. All participants considered in this study had a minimum of 50 trials in each of the six conditions.

Recording and ERP analysis. Signals were recorded with a sampling rate of $256 \mathrm{~Hz}$ using a BIOSEMI Active Two 64-channel EEG system. The reference was set off-line to average electrodes. Epochs including $500 \mathrm{~ms}$ before and after stimulus onset were extracted for further analysis and bandpass filtered with a second-order Butterworth filter between 1 and $70 \mathrm{~Hz}$. Trials with eye movements, blinks, and other artifacts were rejected off-line by visual inspection. After examination of the evoked to-
Have you seen a face?
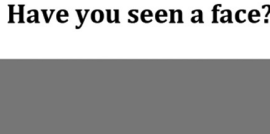

Mask $\mathbf{Y} / \mathbf{N}$
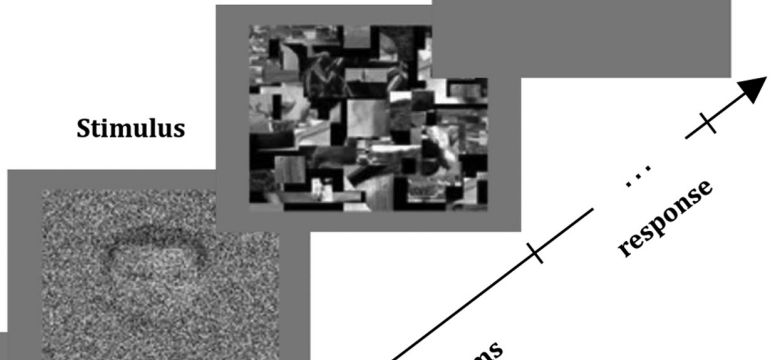
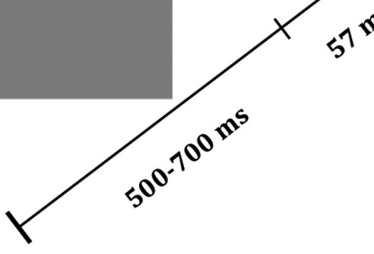

pographies, the PO7 and PO8 electrode sites were selected for further analysis as these showed the best $\mathrm{P} 1, \mathrm{~N} 170$, and $\mathrm{P} 2$ responses. For statistical analysis, we computed for each channel and each subject the mean average ERP between 80 and $120 \mathrm{~ms}$ for the P1, between 150 and $190 \mathrm{~ms}$ for the N170, and between 200 and $240 \mathrm{~ms}$ for the P2 component. For each subject, these values were averaged across channels PO7 and PO8 in each of the six conditions. We then compared the different conditions with a two-factor within-subjects ANOVA with factors "noise level" (NL; three levels: sub-threshold, threshold, or supra-threshold; $\left.\mathrm{df}_{\mathrm{NL}}=2\right)$ and "conscious report" (CR; two levels: face seen or unseen; $\left.\mathrm{df}_{\mathrm{CR}}=1\right)$. The degrees of freedom of the error term for testing the significance of the "noise level" factor were $\mathrm{df}_{\mathrm{NL} \times \mathrm{S}}=\mathrm{df}_{\mathrm{NL}} \times \mathrm{df}_{\mathrm{S}}=2 \times 21=42$. Similarly, the degrees of freedom of the error term for testing the significance of the conscious report factor were $\mathrm{df}_{\mathrm{CR} \times \mathrm{S}}=\mathrm{df}_{\mathrm{CR}} \times \mathrm{df}_{\mathrm{S}}=1 \times 21=21$. Statistical differences were assessed separately for each ERP component (P1, N170, and P2).

Correct rejections of the catch trials (i.e., trials in which we presented a car with noise and the subject reported not seeing a face) were used to calculate a non-face object-evoked response with the level of noise of the threshold condition. To compare the response to cars with the one to seen and unseen faces, we used a one-factor within-subjects ANOVA with main factor "Selectivity" (i.e., Sel, three levels: face seen, face unseen, or car, $\left.\mathrm{df}_{\mathrm{Sel}}=2, \mathrm{df}_{\mathrm{Sel} \times \mathrm{S}}=42\right)$. Post hoc comparisons were performed with Scheffé's tests.

To compare between face and car presentations without noise, we also implemented a one-factor within-subjects ANOVA with main factor "Object" (i.e., Obj, two levels: face and car, $\mathrm{df}_{\mathrm{Obj}}=1, \mathrm{df}_{\mathrm{Obj} \times \mathrm{S}}=21$ ). This analysis was performed to study the face selectivity of the N170 with perfect visibility.

Single-trial analysis. Single-trial ERPs were extracted by means of a denoising algorithm that uses a wavelet decomposition of the single-trial traces and denoises the ERPs by reconstructing the signal using only the wavelet coefficients related to the evoked responses (Quian Quiroga, 2000; Ahmadi and Quian Quiroga, 2013). This method was previously shown to significantly improve the estimation of the single-trial ERPs compared with the original (non-denoised) single-trial traces, as assessed visually with real visual and auditory evoked potentials, and quantita- 

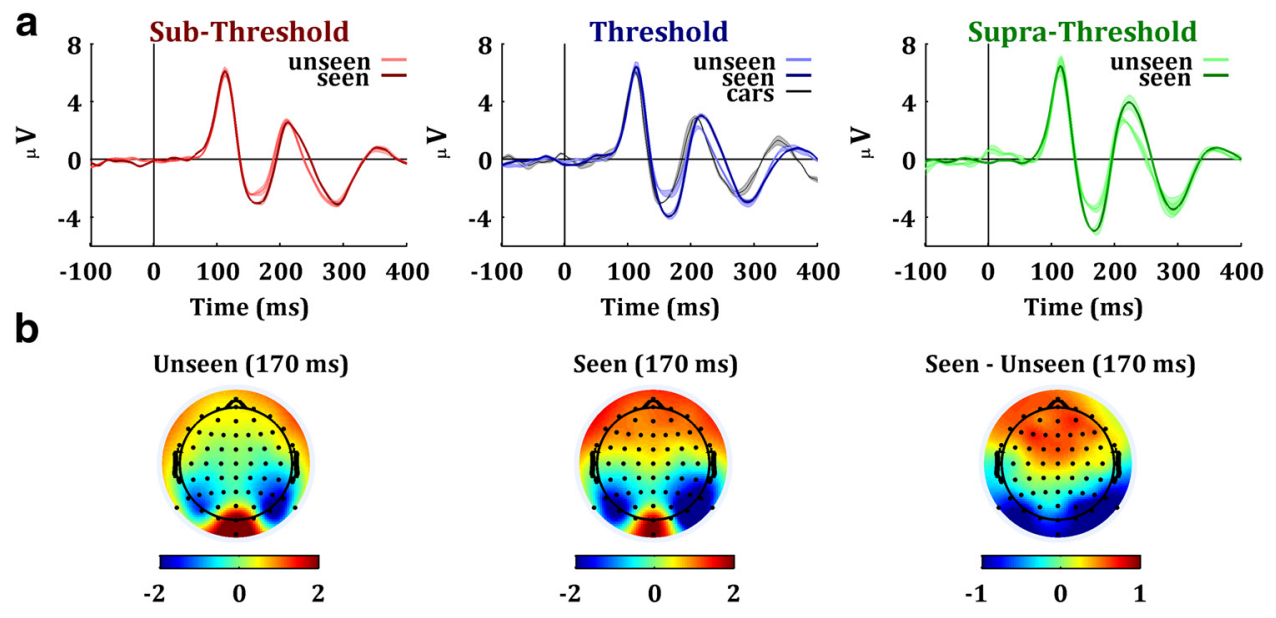

Figure 2. Neural correlates of conscious versus unconscious face perception. $\boldsymbol{a}$, Grand average ERPs recorded at electrode P08 for the sub-threshold, threshold, and supra-threshold conditions. For the threshold condition, we also display the car evoked potential (black line). Bands around mean values denote SEM. $\boldsymbol{b}$, Scalp topography of activation for seen and unseen trials in the threshold condition, 170 ms after stimulus onset. Units of the bottom colorbars are microvolts.

tively with simulated data resembling the components obtained with pattern visual evoked potentials (Quian Quiroga and Garcia, 2003). In the latter case, for different noise levels covering a wide range of signalto-noise ratios found in real recordings, the single-trial denoising significantly decreased the error in the estimation of the single-trial amplitudes and latencies of the different components, compared with the nondenoised data (Quian Quiroga and Garcia, 2003). The set of wavelet coefficients selected to denoise the single-trial data was kept constant for all channels and subjects. The single-trial N170 responses were identified as the local minimum between 120 and $200 \mathrm{~ms}$, and those of the P1 and P2 peaks were identified as the local maximum between $80 \mathrm{~ms}$ and the latency of the N170, and between the N170 peak and $240 \mathrm{~ms}$, respectively. Similar results were obtained with slightly smaller or larger window sizes. To assess the correlation between the single-trial responses and the subjects' report, we implemented a linear discriminant analysis (Fisher, 1936). To evaluate the information provided by each component, in a separate analysis we decoded the behavioral responses by the subjects (face recognized or not) using the values of the $\mathrm{P} 1, \mathrm{~N} 170$, and $\mathrm{P} 2$ peaks in channels PO7 and PO8. For validation of decoding performance, we used a leave-one-out cross-validation. The $p$ value of the decoding performance was estimated by comparing the number of hits to those obtained from a binomial distribution under the assumption of chance guessing (Quian Quiroga and Panzeri, 2009). To further validate the results, statistical significance was also assessed with a nonparametric permutation test. In each permutation, the subjects' reports were shuffled, and the same linear discriminant analysis was implemented. This procedure was repeated 1000 times for each subject and component, thus producing a distribution of number of hits under the null hypothesis. The $p$ value was estimated as the proportion of times that the number of hits from the shuffled distribution exceeded the observed one.

For each condition, the single-trial amplitudes of each component were calculated and latency jitters were computed as the SD of the singletrial latencies of each peak. Statistical differences for the single-trial amplitudes and latency jitters were assessed by the same ANOVA test used for the average evoked responses. Post hoc comparisons were performed using Scheffé's tests.

\section{Results}

\section{Behavior}

The implementation of the double-staircase procedure gave a mean noise variance of $0.45 \pm 0.21$ for the sub-threshold condition; $0.32 \pm 0.17$ for the threshold condition; and $0.20 \pm 0.12$ for the supra-threshold condition. After rejection of trials with blinks, eye-movements, and other artifacts, the proportion of seen trials was $31.3 \pm 7.1 \%$ for the sub-threshold condition;
$49.6 \pm 6.6 \%$ for the threshold condition, and $73.1 \pm 11.8 \%$ for the supra-threshold condition.

\section{Average evoked responses}

We first replicated the existence of the N170 face-selective responses by comparing the ERPs elicited by cars and faces without noise. An ANOVA revealed a significant effect for the N170 $\left(F_{(1,21)}=27.71, p<0.001\right)$, but not for the $\mathrm{P} 1\left(F_{(1,21)}=0.12, p=\right.$ $0.73)$ and the $\mathrm{P} 2\left(F_{(1,21)}=0.55, p=0.47\right)$ components.

To assess the dependence of the N170 with conscious face perception and with the uncertainty introduced by the Gaussian noise, we compared the ERPs for the seen and unseen trials at the three noise levels. Figure 2 shows the grand average ERPs at channel PO8 elicited by these six conditions, as well as the scalp topographies for the seen and unseen faces at the time of the N170 response. The $\mathrm{P} 1$ was neither modulated by conscious face perception nor by noise level. In contrast, we observe a larger N170 and, to a lesser degree, a larger $\mathrm{P} 2$ for the seen trials, especially for the threshold and supra-threshold conditions. Altogether, the responses were also larger for the presentations with a lower noise level (i.e., supra-threshold condition). Statistical analysis revealed that the N170 component presented a significant effect of noise level $\left(F_{(2,42)}=13.93, p<0.001\right)$ and conscious report $\left(F_{(1,21)}=24.92, p<0.001\right)$. The interaction between these factors was not significant $\left(F_{(2,42)}=0.01, p=0.98\right)$. The P1 and P2 components did not show a significant effect of noise level (P1: $\left.F_{(2,42)}=0.3, p=0.74 ; \mathrm{P} 2: F_{(2,42)}=0.91, p=0.41\right)$, conscious report $\left(\mathrm{P} 1: F_{(1,21)}=1.14, p=0.29 ; \mathrm{P} 2: F_{(1,21)}=2.55, p=0.12\right)$ or interaction $\left(\mathrm{P} 1: F_{(2,42)}<0.01, p>0.99 ; \mathrm{P} 2: F_{(2,42)}<0.01, p>\right.$ $0.99)$.

We also compared the activity elicited by cars with the one by the seen and unseen faces at the threshold condition (Fig. 2a). An ANOVA showed a significant effect of selectivity $\left(F_{(2,42)}=8.18\right.$, $p<0.001)$. Post hoc comparisons with Scheffé's tests revealed that the N170 elicited by cars was not significantly different to the one triggered by the unseen faces $\left(t_{(21)}=2.7<t_{\text {scheffé }}=4.04\right)$ and was significantly smaller than the one for the seen faces $\left(t_{(21)}=4.2>\right.$ $\left.t_{\text {scheffé }}=4.04, p<0.001\right)$.

\section{Single-trial analysis}

Differences observed in the average responses could be due to different mechanisms. For instance, an increase of the average 
a
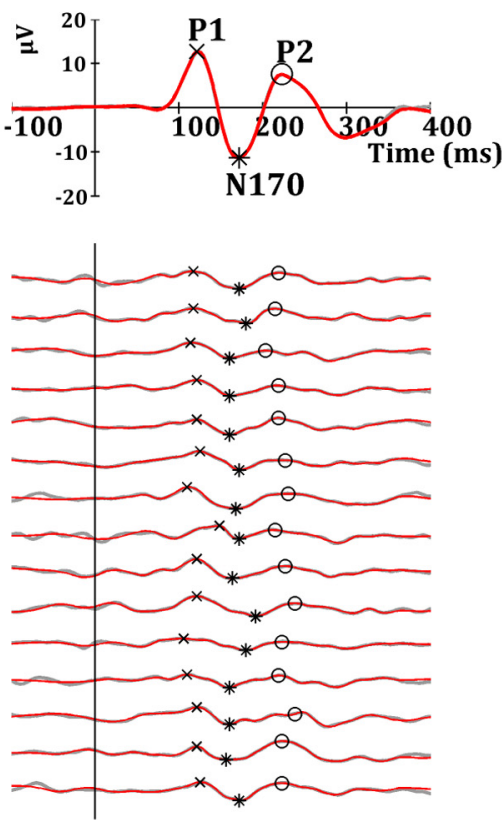

b
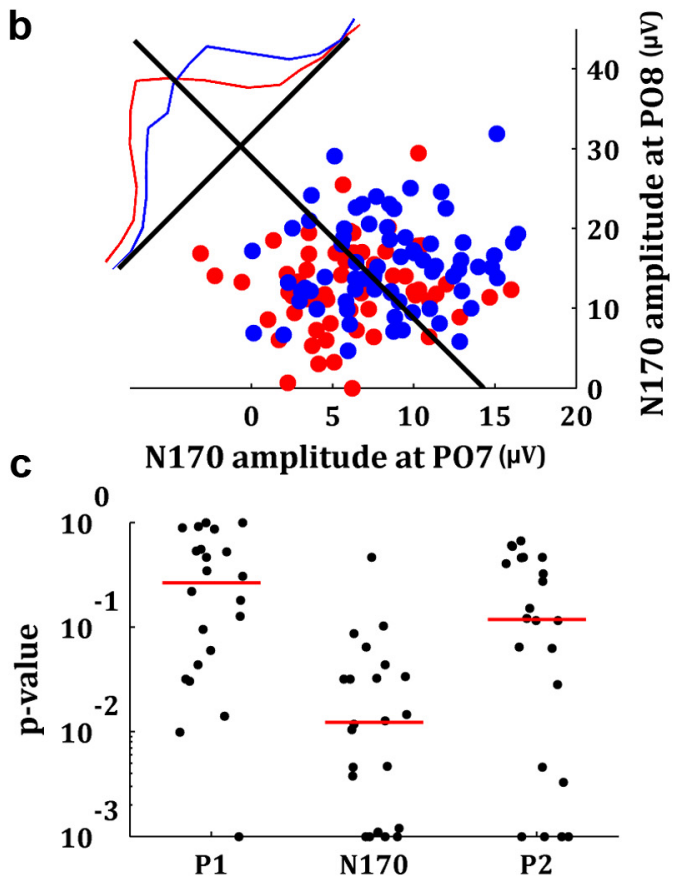

Figure 3. Decoding of the conscious reports with the single-trial N170. $a$, Average ERP and 15 denoised single-trial ERPs at electrode P08 for a typical subject. The identification of the single-trial peaks is shown with the markers. $\boldsymbol{b}$, Distribution of single-trial N170 responses in a typical participant used for decoding. Each blue (red) dot represents the single-trial N170 amplitudes measured at electrodes P07 and P08 in a seen (unseen) trial. The black line represents the Fisher's linear discriminant. The blue and red lines show the normalized distributions for seen and unseen trials projected along the axis perpendicular to the linear discriminant. c, Black dots show in a logarithmic scale the individual $p$ values of the decoding performance with different components. The red lines indicate the median of the distribution of $p$ values for each component.

ERP could be due to an increase of the amplitude of the singletrial responses, or due to a better time locking to the onset of the stimulus, which reduces the latency jitter. To distinguish between these possibilities, we implemented a single-trial analysis. The single-trial P1, N170, and P2 peaks were extracted by identifying the local maxima (minima) of the denoised responses in different time windows (Fig. $3 a$; see Materials and Methods). The singletrial amplitude of the N170 was significantly larger for the trials in which the faces were recognized $\left(F_{(1,21)}=64.12, p<0.001\right)$. In contrast, this difference was not significant for the $\mathrm{P} 1\left(F_{(1,21)}=\right.$ $0.09, p=0.75)$ and the $\mathrm{P} 2\left(F_{(1,21)}=2.1, p=0.13\right)$ components. Post hoc comparisons revealed that the differences between seen and unseen trials in the N170 component were significant for the threshold $\left(t_{(21)}=9.7>t_{\text {scheffé }}=8.01, p<0.001\right)$ and suprathreshold $\left(t_{(21)}=9.74>t_{\text {scheffé }}=8.01, p<0.001\right)$ conditions, but not for the sub-threshold condition $\left(t_{(21)}=2.25<t_{\text {scheffé }}=\right.$ 8.01).

Given the single-trial amplitude differences for the N170 responses, we examined whether we could use this feature to decode the participants' report (face seen or unseen). For this, we used a linear discriminant analysis with two features (single-trial $\mathrm{N} 170$ at the electrodes PO7 and PO8) and a leave-one-out crossvalidation. Figure $3 b$ shows the single-trial N170 amplitude for one typical subject at the two electrodes selected for decoding. Decoding performance was significantly higher than chance for most subjects $(18 / 22$ with $p<0.05)$, with a mean decoding performance of $60.1 \%$ (SD $4.2 \%$ ). In contrast, using the P1 and P2 components, for most subjects (16/22 in both cases), the decoding performance was at chance level $(\mathrm{P} 1$ : mean $=50.4 \%, \mathrm{SD}=$ $11.0 \%$; 2 : mean $=54.8 \%, \mathrm{SD}=6.3 \%)$. The median of the distribution of $p$ values was 0.01 for the N170, 0.26 for the P1, and 0.11 for the $\mathrm{P} 2$ components (Fig. 3 c). The nonparametric permutation test (see Materials and Methods) gave very similar results
(Median of $p$ value distributions: 0.01 for the N170, 0.28 for the $\mathrm{P} 1$, and 0.16 for the $\mathrm{P} 2$ components).

Next, we studied the single-trial responses for the different noise levels. Figure 4 shows the mean and SD of the single-trial N170 amplitude (Fig. 4a) and latency jitter (Fig. 4b) at channel PO8 for all conditions. Similar results were obtained with electrode site PO7. Despite the differences seen in the average responses, an ANOVA test showed that the differences in the single-trial amplitudes for the three noise levels were not significant for any component: $\mathrm{N} 170\left(F_{(2,42)}=2.15, p=0.13\right)$; P1 $\left(F_{(2,42)}=0.35, p=0.71\right)$; P2 $\left(F_{(2,42)}=0.55, p=0.58\right)$. The interaction between noise level and conscious report was also not significant for the $\mathrm{N} 170\left(F_{(2,42)}=0.03, p=0.97\right), \mathrm{P} 1\left(F_{(2,42)}=\right.$ $0.01, p=0.99)$, and P2 $\left(F_{(2,42)}=0.02, p=0.98\right)$. For the N170, the lack of a difference in the single-trial amplitudes, but the significant amplitude difference in the average, suggested the presence of different degrees of latency variability for the three noise conditions. As expected, we found a significant negative correlation between the N170 latency jitter and the mean amplitude of the grand average $\mathrm{N} 170(r=-0.98, p=0.002)$. Moreover, an ANOVA revealed that the N170 latency jitter was significantly modulated by noise level $\left(F_{(2,42)}=4.96, p=0.01\right)$ but not by conscious report $\left(F_{(1,21)}=0.9, p=0.35\right)$. Post hoc comparisons revealed that the modulation by noise level was explained by a significant difference between the sub-threshold and supra-threshold condition $\left(t_{(21)}=3.21>t_{\text {scheffé }}=3.14, p<\right.$ $0.01)$. Differences in latency jitter were not significant for the P1 (noise level: $F_{(2,42)}=1.83, p=0.17$; conscious report: $F_{(1,21)}=$ $1.24, p=0.27$ ) and the P2 (noise level: $F_{(2,42)}=2.55, p=0.09$; conscious report: $\left.F_{(1,21)}=1.62, p=0.21\right)$ components. The mean latency of the N170 was modulated neither by conscious recognition $\left(F_{(1,21)}=0.62, p=0.44\right)$ nor by noise level $\left(F_{(2,42)}=\right.$ 2.23, $p=0.12)$, thus indicating that the stimulus uncertainty 
a

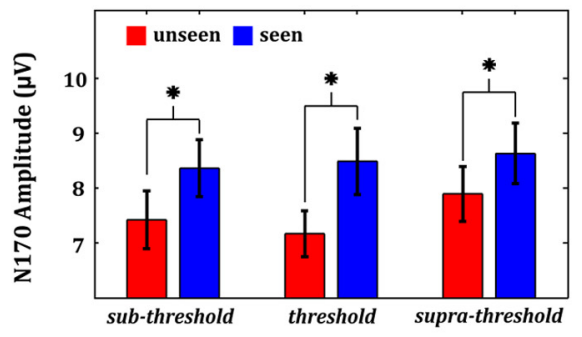

b

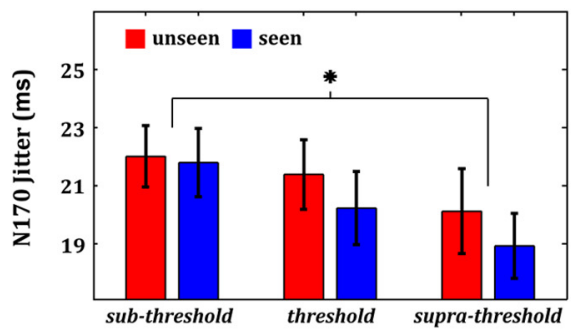

C
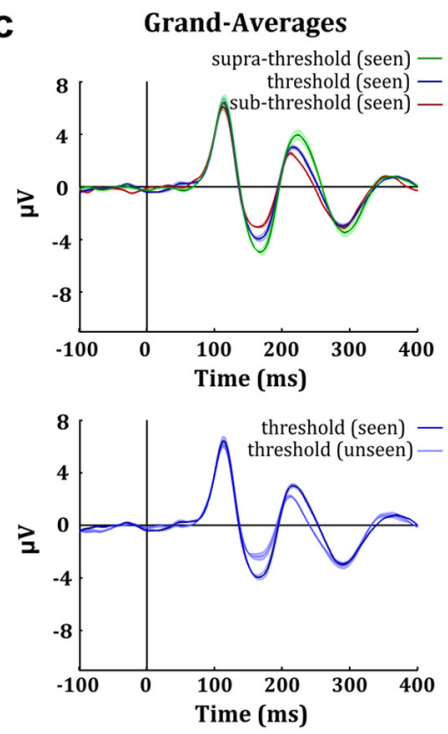

Latency-Corrected
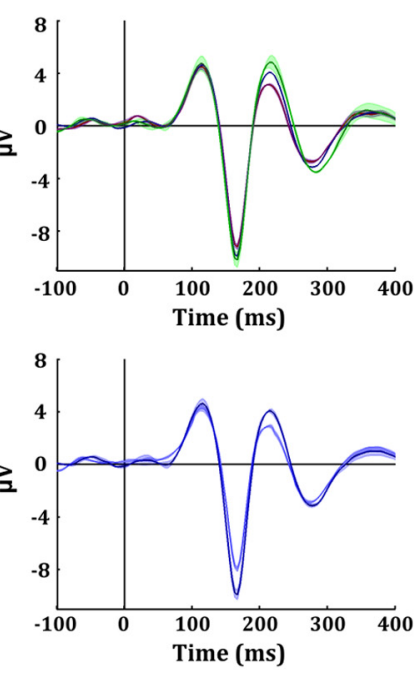

Figure 4. Single-trial analysis of ERP responses. $\boldsymbol{a}$, Mean and SD of the single-trial N170 amplitude at channel P08. $\boldsymbol{b}$, Mean and SD of the N170 latency jitter at channel P08. $\boldsymbol{c}$, The noise-level effect (electrode site: P08) measured at the average ERP level (top-left panel) vanished after latency-correction (top-right panel). In contrast, the conscious-report effect (bottom-left panel) persisted after latency correction (bottom-right panel). Bands around mean values denote SEM.

introduced by the noise modulated the timing consistency across trials but not the mean timing of occurrence of the N170 responses.

The single-trial analysis also allowed us to remove latency jitters and to compute latency-corrected averages - i.e., averages in which each single trial is shifted in time to have the same latency as the average response. Figure $4 c$ shows the grand average and the latency-corrected grand average ERPs at channel PO8 for the three conditions in which subjects reported the presence of a face. In line with the findings described above, the noise-level effect vanished after latency correction of the N170, and the differences between the different noise levels became nonsignificant $\left(F_{(2,42)}=1.77, p=0.18\right)$. In contrast, the latency-corrected N170 still exhibited a significant modulation by conscious report (Fig. $\left.4 c, F_{(1,21)}=8.82, p=0.007\right)$. In summary, conscious face perception modulated the single-trial amplitudes of the N170, whereas the uncertainty introduced by the Gaussian noise increased the variability of the N170 latency across trials, thus giving a reduced peak in the average ERP.

\section{Discussion}

The neural correlates of object recognition have been explored with different brain signals, going from EEG (Lamy et al., 2009; Fabre-Thorpe, 2011; Martens et al., 2011), MEG (Liu et al., 2002; Tanskanen et al., 2007), fMRI (Malach et al., 1995; Grill-Spector et al., 2000; Bar et al., 2001), and electrocorticography (ECoG) (Fisch et al., 2009; Gaillard et al., 2009), to single-cell recordings in human patients (Quian Quiroga et al., 2008) and nonhuman primates (for review, see Macknik, 2006). Among the subset of works that focused on face processing, there is an agreement that the inferotemporal cortex around the fusiform gyrus plays a decisive role in face recognition. This area is known to display faceselective responses in single-cell recordings (Tsao et al., 2006) as well as during noninvasive imaging (Kanwisher et al., 1997), and it was shown to be the source of the evoked N170 component measured with EEG recordings (Shibata et al., 2002).

Several works have studied the N170 in response to face presentations compared with other stimuli (Eimer, 2011). In line with these works, in our study we explored whether the N170 responses were modulated by conscious face perception and by the stimulus uncertainty introduced by different levels of noise. Using identical visual stimuli, we found that the amplitude of the average N170 was larger for recognized than for nonrecognized faces. Moreover, the average N170 diminished with increasing levels of noise. These two effects were specific for the N170 as they were absent in earlier (P1) and later (P2) ERP components. Furthermore, we investigated the mechanisms underlying these modulations by analyzing the single-trial amplitudes and latencies of the different components.

In general, ERPs are very small compared with the ongoing EEG oscillations, and their analysis relies on the identification of different peaks after averaging several trials. Although averaging improves the signal-to-noise ratio, important information about single-trial variations is completely dismissed, despite the fact that trial-to-trial changes can provide useful information about the mechanisms underlying the modulations observed in the average responses (Quian Quiroga et al., 2007). For instance, sleepdependent enhancements of the average mismatch negativity-a component observed in auditory oddball paradigms-that were at first thought to be due to a recruitment of larger populations of neurons during sleep (Atienza et al., 2004), were later found to be due to a completely different mechanism after a single-trial analysis, namely, a decrease in the latency jitter of the single-trial responses (Atienza et al., 2005). Similar to the effect reported in that study, here we showed that the stimulus uncertainty introduced by the Gaussian noise decreased the average N170 by impoverishing the timing consistency (i.e., increasing the latency jitter) but not the amplitude of the single-trial responses. In turn, the facerecognition effect observed in the average N170 was linked to an attenuation of the single-trial responses for the unseen trials.

Previous studies have also implemented a single-trial approach to study the face versus non-face object sensitivity of the N170 (Philiastides and Sajda, 2006; Rousselet et al., 2007; Gaspar et al., 2011). For instance, it was found that this effect is correlated with an increase of the single-trial power at the $5-15 \mathrm{~Hz}$ band (Rousselet et al., 2007). Moreover, a single-trial decoding approach was also used to distinguish between face and object pre- 
sentations with different levels of uncertainty (Philiastides and Sajda, 2006). While these works were focused on the face versus non-face object modulation, here we studied the single-trial underpinnings of the face-recognition effect (i.e., the difference between seen and unseen faces). Interestingly, it was recently shown that N170 grand averages might be misleading, suggesting that a single-subject analysis is essential to a correct interpretation of the results (Gaspar et al., 2011). Our study provides further evidence along this line, showing that even more information can be extracted from the single-trial analysis of the responses.

Most of the previous studies linking the average N170 to conscious face perception compared the activity elicited by "recognized" versus "not-recognized" stimuli inducing the different perceptual outcomes by changing physical properties of the stimuli (Jemel et al., 2003; Liddell et al., 2004; Bacon-Macé et al., 2005; Genetti et al., 2009; Pegna et al., 2011). The caveat of this approach is that it mixes up the effect of conscious recognition with the one given by processing different types of stimuli. In contrast, in this work the effect of conscious face perception was assessed by comparing the activity elicited by identical stimuli.

Only few previous works have used identical stimuli to study the N170 modulation by conscious face perception (Fisch et al., 2009; Genetti et al., 2010; Rodríguez et al., 2012). Genetti et al. (2010) reported differences due to face recognition but only at latencies larger than the one of the N170, probably due to the use of very short stimulus durations (16 ms). Another recent work (Rodríguez et al., 2012) showed that only fully perceived faces have an N170 activity significantly stronger than scrambled faces, while the activity of unseen faces was consistent with the one elicited by scrambled faces (though in this work seen and unseen faces were not directly compared with each other). In line, here we showed that seen, but not unseen, faces elicit an N170 significantly larger than cars. To the best of our knowledge, only one previous ECoG study, which focused on the analysis of gammainduced oscillations, reported differences in the N170 elicited by seen and unseen faces using identical stimuli (Fisch et al., 2009). Building on the results of this study, here we described further evidence about the relationship of the N170 component with conscious face processing by showing that: (1) the intracranial responses observed by Fisch et al. (2009) are observable at the level of the scalp EEG, which has been by far the most used signal to study N170 in face perception; (2) this effect was specific to the $\mathrm{N} 170$ as it was absent in earlier and later components (the P1 and $\mathrm{P} 2$, respectively); (3) at the single-trial level the N170 could predict the behavioral responses better than chance; and (4) two different mechanisms underlie the modulation of the N170 responses with conscious recognition and with the stimulus uncertainty introduced with the Gaussian noise.

Summarizing, the current study provides evidence that the average N170 is modulated by conscious face perception, as well as by the amount of noise added to the stimulus. However, these two effects modulate the average N170 through different mechanisms, as shown by the single-trial analysis. While conscious face perception was correlated with a boost in the activity of faceselective neural assemblies, the stimulus uncertainty introduced by the Gaussian noise induced a decrease in the timing consistency (but not in the amplitude) of this activation.

\section{References}

Ahmadi M, Quian Quiroga R (2013) Automatic denoising single-trial evoked potentials. Neuroimage 66:672-680. CrossRef Medline

Atienza M, Cantero JL, Stickgold R (2004) Posttraining sleep enhances automaticity in perceptual discrimination. J Cogn Neurosci 16:53-64. CrossRef Medline
Atienza M, Cantero JL, Quian Quiroga R (2005) Precise timing accounts for posttraining sleep-dependent enhancements of the auditory mismatch negativity. Neuroimage 26:628-634. CrossRef Medline

Bacon-Macé N, Macé M, Fabre-Thorpe M, Thorpe SJ (2005) The time course of visual processing: backward masking and natural scene categorisation. Vision Res 45:1459-1469. CrossRef Medline

Bar M, Tootell RB, Schacter DL, Greve DN, Fischl B, Mendola JD, Rosen BR, Dale AM (2001) Cortical mechanisms specific to explicit visual object recognition. Neuron 29:529-535. CrossRef Medline

Bentin S, Allison T, Puce A, Perez E, McCarthy G (1996) Electrophysiological studies of face perception in humans. J Cogn Neurosci 8:551-565. CrossRef Medline

Eimer M (2011) The face-sensitive N170 component of the event-related brain potential. In: The oxford handbook of face perception (Calder AJ, Rhodes G, Johnson M, Haxby J, eds), pp 329-344. Oxford, UK: Oxford UP.

Engell AD, McCarthy G (2010) Selective attention modulates face-specific induced gamma oscillations recorded from ventral occipitotemporal cortex. J Neurosci 30:8780-8786. CrossRef Medline

Fabre-Thorpe M (2011) The characteristics and limits of rapid visual categorization. Front Psychol 2:243. CrossRef Medline

Fisch L, Privman E, Ramot M, Harel M, Nir Y, Kipervasser S, Andelman F, Neufeld MY, Kramer U, Fried I, Malach R (2009) Neural "ignition": enhanced activation linked to perceptual awareness in human ventral stream visual cortex. Neuron 64:562-574. CrossRef Medline

Fisher RA (1936) The use of multiple measurements in taxonomic problems. Ann Hum Genet 7:179-188.

Furey ML, Tanskanen T, Beauchamp MS, Avikainen S, Uutela K, Hari R, Haxby JV (2006) Dissociation of face-selective cortical responses by attention. Proc Natl Acad Sci U S A 103:1065-1070. CrossRef Medline

Gaillard R, Dehaene S, Adam C, Clémenceau S, Hasboun D, Baulac M, Cohen L, Naccache L (2009) Converging intracranial markers of conscious access. PLoS Biol 7:e61. CrossRef Medline

Gaspar CM, Rousselet GA, Pernet CR (2011) Reliability of ERP and singletrial analyses. Neuroimage 58:620-629. CrossRef Medline

Gauthier I, Curran T, Curby KM, Collins D (2003) Perceptual interference supports a non-modular account of face processing. Nat Neurosci 6:428 432. CrossRef Medline

Genetti M, Khateb A, Heinzer S, Michel CM, Pegna AJ (2009) Temporal dynamics of awareness for facial identity revealed with ERP. Brain Cogn 69:296-305. CrossRef Medline

Genetti M, Britz J, Michel CM, Pegna AJ (2010) An electrophysiological study of conscious visual perception using progressively degraded stimuli. J Vis 10(14):10 1-14. CrossRef Medline

Grill-Spector K, Kushnir T, Hendler T, Malach R (2000) The dynamics of object-selective activation correlate with recognition performance in humans. Nat Neurosci 3:837-843. CrossRef Medline

Harris JA, Wu C, Woldorff MG (2011) Sandwich masking eliminates both visual awareness of faces and face-specific brain activity through a feedforward mechanism. J Vis 11(7):3 1-12. CrossRef Medline

Jemel B, Schuller AM, Cheref-Khan Y, Goffaux V, Crommelinck M, Bruyer R (2003) Stepwise emergence of the face-sensitive N170 event-related potential component. Neuroreport 14:2035-2039. CrossRef Medline

Kanwisher N, McDermott J, Chun MM (1997) The fusiform face area: a module in human extrastriate cortex specialized for face perception. J Neurosci 17:4302-4311. Medline

Lamy D, Salti M, Bar-Haim Y (2009) Neural correlates of subjective awareness and unconscious processing: an ERP study. J Cogn Neurosci 21: 1435-1446. CrossRef Medline

Landau AN, Esterman M, Robertson LC, Bentin S, Prinzmetal W (2007) Different effects of voluntary and involuntary attention on EEG activity in the gamma band. J Neurosci 27:11986-11990. CrossRef Medline

Liddell BJ, Williams LM, Rathjen J, Shevrin H, Gordon E (2004) A temporal dissociation of subliminal versus supraliminal fear perception: an eventrelated potential study. J Cogn Neurosci 16:479-486. CrossRef Medline

Liu J, Harris A, Kanwisher N (2002) Stages of processing in face perception: an MEG study. Nat Neurosci 5:910-916. CrossRef Medline

Macknik SL (2006) Visual masking approaches to visual awareness. In: Visual perception, Pt 2, Vol 155: fundamentals of awareness: multi-sensory integration and high-order perception (Martinez-Conde S, Macknik S, Martinez MM, Alonso J-M, Tse PU, eds), pp 177-215. New York: Elsevier. Malach R, Reppas JB, Benson RR, Kwong KK, Jiang H, Kennedy WA, Ledden 
PJ, Brady TJ, Rosen BR, Tootell RB (1995) Object-related activity revealed by functional magnetic-resonance-imaging in human occipital cortex. Proc Natl Acad Sci U S A 92:8135-8139. CrossRef Medline

Martens U, Trujillo-Barreto N, Gruber T (2011) Perceiving the tree in the woods: segregating brain responses to stimuli constituting natural scenes. J Neurosci 31:17713-17718. CrossRef Medline

McKone E, Kanwisher N, Duchaine BC (2007) Can generic expertise explain special processing for faces? Trends Cogn Sci 11:8-15. CrossRef Medline

Pegna AJ, Darque A, Berrut C, Khateb A (2011) Early ERP modulation for task-irrelevant subliminal faces. Front Psychol 2:88. CrossRef Medline

Philiastides MG, Sajda P (2006) Temporal characterization of the neural correlates of perceptual decision making in the human brain. Cereb Cortex 16:509-518. CrossRef Medline

Quian Quiroga R (2000) Obtaining single stimulus evoked potentials with wavelet denoising. Physica D 145:278-292. CrossRef

Quian Quiroga R, Garcia H (2003) Single-trial event-related potentials with wavelet denoising. Clin Neurophysiol 114:376-390. CrossRef Medline

Quian Quiroga R, Panzeri S (2009) Extracting information from neuronal populations: information theory and decoding approaches. Nat Rev Neurosci 10:173-185. CrossRef Medline

Quian Quiroga R, Atienza M, Cantero JL, Jongsma M (2007) What can we learn from single-trial event-related potential. Chaos Complexity Lett 2:345-363.

Quian Quiroga R, Mukamel R, Isham EA, Malach R, Fried I (2008) Human single-neuron responses at the threshold of conscious recognition. Proc Natl Acad Sci U S A 105:3599-3604. CrossRef Medline
Rodríguez V, Thompson R, Stokes M, Brett M, Alvarez I, Valdes-Sosa M, Duncan J (2012) Absence of face-specific cortical activity in the complete absence of awareness: converging evidence from functional magnetic resonance imaging and event-related potentials. J Cogn Neurosci 24:396-415. CrossRef Medline

Rossion B, Jacques C (2011) The N170: understanding the time-course of face perception in the human brain. In: The oxford handbook of ERP components (Luck S, Kappenman E, eds), pp 115-142. Oxford, UK: Oxford UP.

Rossion B, Gauthier I, Goffeaux V, Tarr MJ, Crommelinck M (2002) Expertise training with novel objects leads to left-lateralized facelike electrophysiological responses. Psychological Science 13:250-257. CrossRef Medline

Rousselet GA, Husk JS, Bennett PJ, Sekuler AB (2007) Single-trial EEG dynamics of object and face visual processing. Neuroimage 36:843862. CrossRef Medline

Shibata T, Nishijo H, Tamura R, Miyamoto K, Eifuku S, Endo S, Ono T (2002) Generators of visual evoked potentials for faces and eyes in the human brain as determined by dipole localization. Brain Topogr 15:5163. CrossRef Medline

Tanskanen T, Näsänen R, Ojanpää H, Hari R (2007) Face recognition and cortical responses: effect of stimulus duration. Neuroimage 35 : 1636-1644. CrossRef Medline

Tsao DY, Freiwald WA, Tootell RB, Livingstone MS (2006) A cortical region consisting entirely of face-selective cells. Science 311:670-674. CrossRef Medline 